\title{
Far-Field Boundary Conditions for Calculation of Hole-Drilling Residual Stress Calibration

\author{
Coefficients
}

\author{
Antonio Baldi \\ Dipartimento di Ingegneria Meccanica, Chimica e dei Materiali \\ Università degli Studi di Cagliari \\ Via Marengo, 2, I-09123 Cagliari, Italy \\ e-mail:antonio.baldi@dimcm. unica.it
}

\section{Abstract/Introduction}

The Hole-Drilling method for residual stress measurement, both in its standard version based on strain gauge rosettes [1] and its derivative using optical methods for estimating the displacement field around the hole [2 4], relies on numerical calibrated coefficients (A and B) to correlate the experimentally acquired strains (displacements) with residual stress components.

To estimate the A and B coefficients, two FEM (Finite Element Method) computations are required, the former related to a hydrostatic stress state, the latter to a pure shear case. Both can be implemented using either a semianalytical approach (i.e. an axis-symmetric mesh expanded in the tangential direction using a Fourier series) or a tri-dimensional mesh, usually exploiting the double symmetry of the problem. 
Whatever the approach selected, the definition of constraints to be applied to the outer boundary is critical because the hole-drilling method assumes an infinite plate, thus both the usual solutions - fully constrained or free boundariesare unable to correctly describe the theoretical situation.

In the following, the problem of correct simulation of the infinite domain will be discussed and two simple and effective solutions will be proposed.

Keywords: Residual Stress, Hole Drilling, Calibration Coefficients, Finite Element Analysis, Boundary Conditions

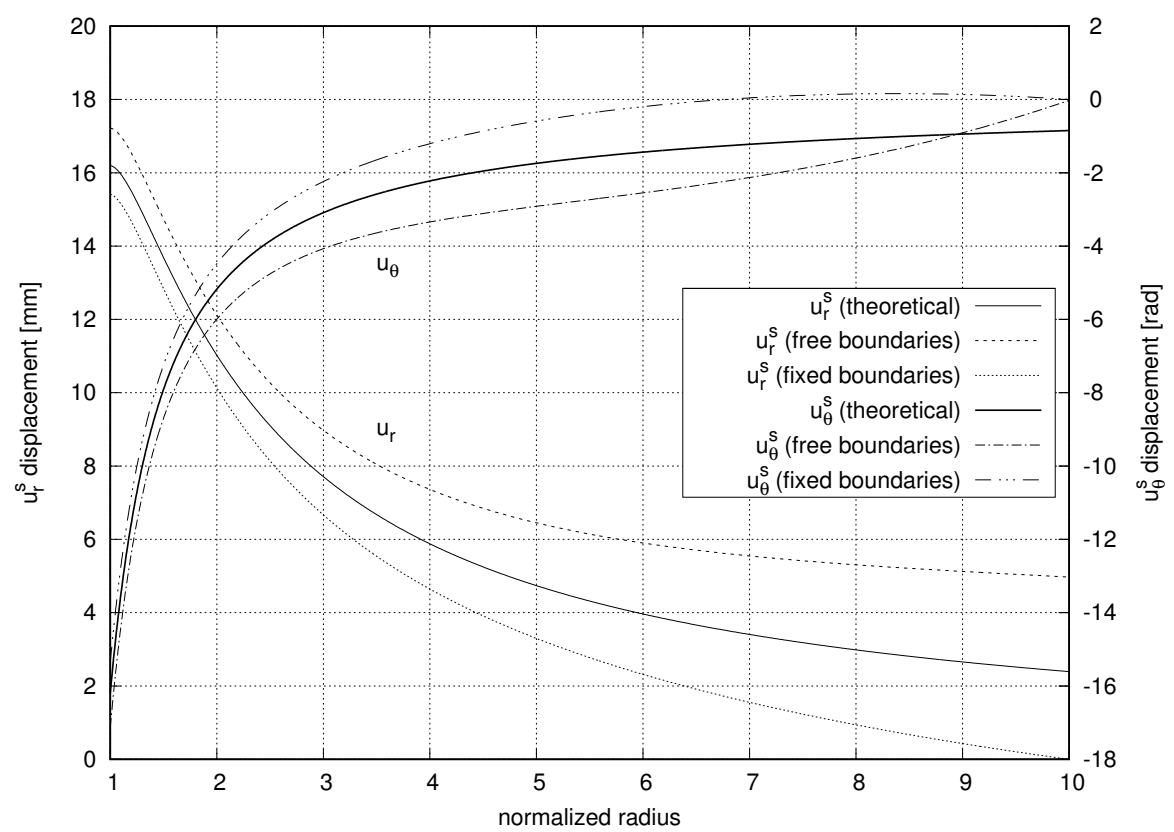

Figure 1: Comparing the theoretical result (hole through case) and the FEM solution with either constrained or free external boundary. Shear-loading case. Displacements computed using a semi-analytical solver (i.e. applying an axissymmetric solution expanded in Fourier series), thus, the tangential displacements correspond to $\theta=\pi / 4$, whereas the radial results are related to $\theta=0$. 


\section{Meshing an Infinite Plate}

The displacement field around a hol $\oint^{1}$ can be written as

$$
\begin{gathered}
u_{r}=A\left(\sigma_{x}+\sigma_{y}\right)+B\left[\left(\sigma_{x}-\sigma_{y}\right) \cos (2 \theta)+2 \tau_{x y} \sin (2 \theta)\right] \\
u_{\theta}=C\left[\left(\sigma_{x}-\sigma_{y}\right) \sin (2 \theta)-2 \tau_{x y} \cos (2 \theta)\right] \\
u_{z}=F\left(\sigma_{x}+\sigma_{y}\right)+G\left[\left(\sigma_{x}-\sigma_{y}\right) \cos (2 \theta)+2 \tau_{x y} \sin (2 \theta)\right]
\end{gathered}
$$

where the $A, B, C, F$ and $G$ coefficients depend on material properties (Young's module $E$ and the Poisson ratio $\nu$ ), on geometric parameters (the hole radius $a$ and the plate thickness $t$ ) and on point location (the ratio of the hole radius to the distance of the current point from the center of the hole $\rho$ and the angular coordinate $\theta$ ). In the blind-hole case, the $A \ldots G$ coefficients have to be computed numerically, but a theoretical solution is known for the through-hole case [5]:

$$
\begin{gathered}
A=\frac{a}{2 E}(1+\nu) \rho \quad B=\frac{a}{2 E}\left[4-(1+\nu) \rho^{2}\right] \rho \\
C=-\frac{a}{2 E}\left[2(1-\nu)+(1+\nu) \rho^{2}\right] \rho \quad F=0 \quad G=\frac{\nu t}{E} \rho^{2}
\end{gathered}
$$

thus, it can be used as a reference to check the accuracy of the numerical solution. Figure 1 compares the FEM-computed radial and tangential displacements assuming either fully constrained or free external boundaries with $1 \mathrm{a}$ and $1 \mathrm{~b}$ (parameters: $\sigma_{x}=-\sigma_{y}=1 \mathrm{MPa}, \tau_{x y}=0, E=1 \mathrm{MPa}, a=6 \mathrm{~mm}, \nu=0.3$, outer diameter $b=10 a$ ). Observing Figure 1 , it is apparent that both solutions differ significantly from the theoretical one; indeed, the former implies that the stiffness of the outer region is infinite, whereas the latter lacks its contribution to overall stiffness (note that the same behavior can also be observed in the hydrostatic loading case).

\footnotetext{
${ }^{1}$ It is worth noting that the same formulas are used in the ring-core method; thus the following discussion also applies to the evaluation of calibration coefficients for this technique.
} 
Apparently, a simple solution to this problem exists in literature: it suffices to add an extra ring of 'infinite' elements to the mesh [6]; indeed, the radial shape function of an infinite element approximates a sequence of the decay form $C_{1} / \zeta+C_{2} / \zeta^{2}+\ldots$, where $\zeta$ is the distance from the focus of the problem, thus allowing correct description of the behavior of the outer part of the plane. Using this solution, a finite segment $A B$ is mapped to $[A: \infty)$, where point $A$ is located midway between the pole of the expansion and $B$; thus, the location of the focus is implicitly defined by the radial coordinate of points $A$ and $B$.

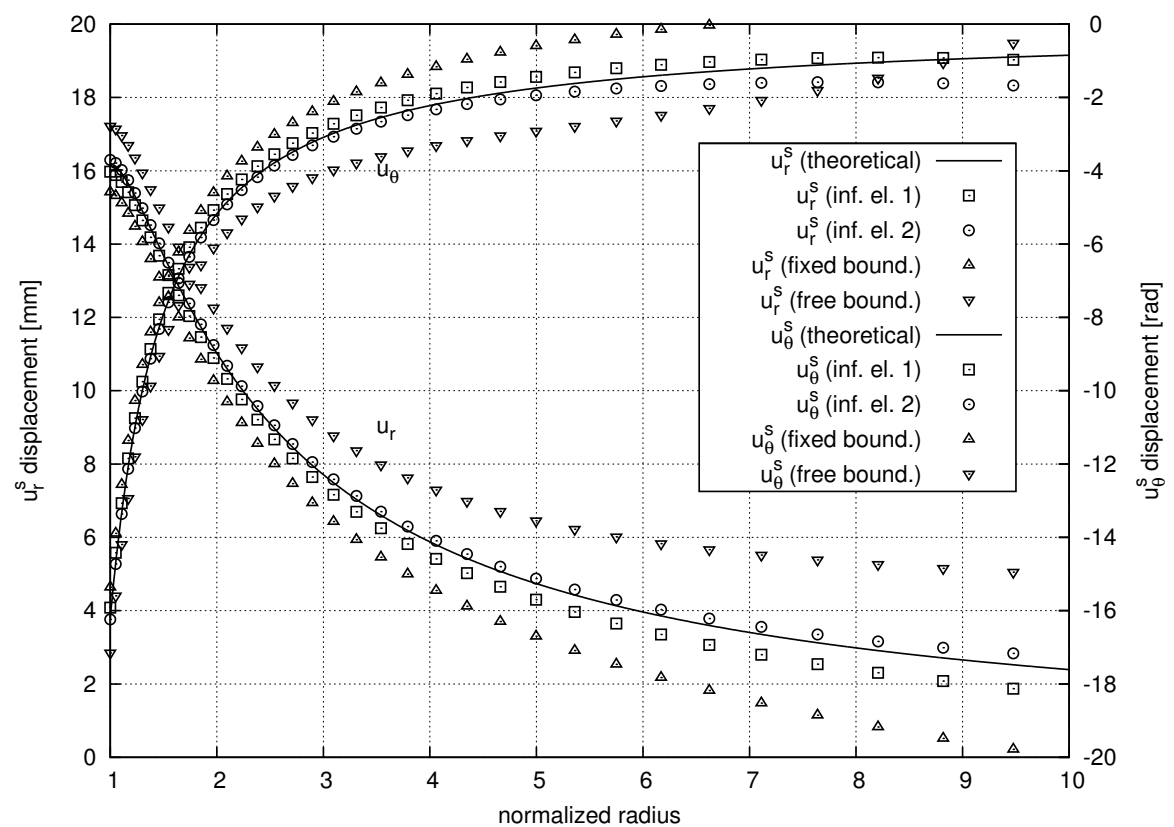

Figure 2: Comparison of FEM solutions with respect to theoretical behavior when using infinite elements (shear-loading case). Two formulations were tested: the first version assumed that the pole of radial expansion was located on the axis of symmetry while in the second version pole location was adjusted to have a congruent normalized radius at $b$. Similar behavior is observed in the hydrostatic case (results of simulation were better, but an exact match was not obtained).

Because calibration coefficients (2) vanish as $\rho=a / r$, two different options 
for the radial location of the pole have been tested (Figure 2): the former (inf. el. 1) assumes the pole is located on the axis of symmetry (thus ring width is equal to $b$ ), the latter (inf. el. 2) ensures that the value of $\zeta$ matches $\rho$ at the interface (i.e. ring width is $b / a$ ). Both solutions outperform the "standard" ones, however, (as noted in [6]) "miracles should not be expected" and the solutions computed using this approach, although better than previous ones, do not exactly match the theoretical behavior 2

\section{A Simple Solution: Using a Fit Ring}

Schajer ${ }^{3}$ suggested a simple solution to the problem of the missing portion of the infinite plane: that of adding a thin ring of high-stiffness elements outside the "standard" mesh in such a way as to have the same radial stiffness as the theoretical infinite material in the far-field. The suggested Young's module is $E_{1}=E b /[t(1+\nu)]$ whereas ring width has to be estimated numerically.

After calibration, the proposed approach ensures exact estimation of radial displacement components in the hydrostatic case ( $A$ and $F$ terms). However, the shear case ( $B, C$ and $G$ coefficients) requires two degrees of freedom, because we have to match both the radial and tangential behavior of the plane in the far field, while we have only one parameter to adjust. It is obviously possible to obtain exact radial displacements but at the expense of the accuracy of tangential displacements. Here we propose an alternative approach: that of fitting at the same time the radial and torsional stiffness of the far field material. To this end we have to adjust both Young's modulus and the width of the ring.

\footnotetext{
${ }^{2}$ Note that infinite elements are not always available in commercial codes.

${ }^{3}$ Gary S. Schajer, personal communication.

${ }^{4}$ Actually, quite satisfactory results are obtained using the same width as in the hydrostatic case.
} 


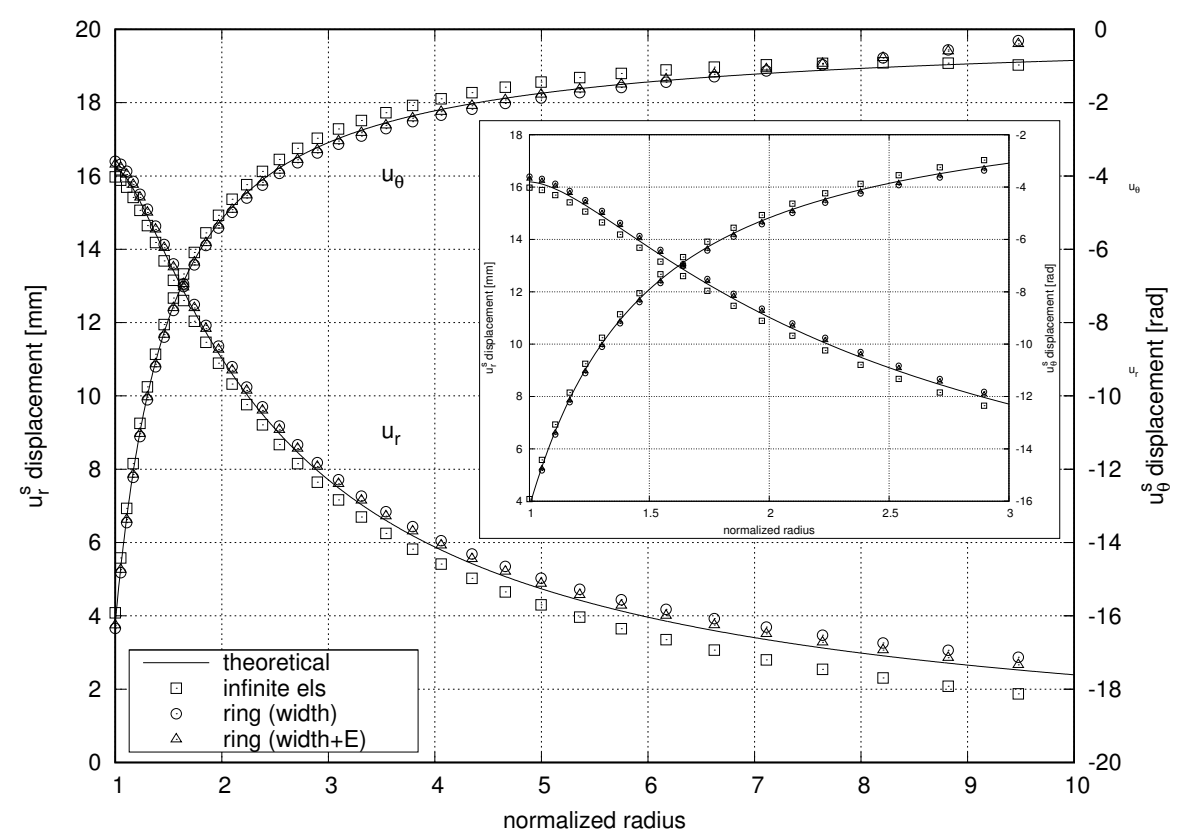

Figure 3: Comparison of FEM solutions with respect to theoretical behavior when using infinite elements, width-only fit-ring and width $+E$ fit-ring. Shearloading case. In the inset: a detail of the area near the hole.

\subsection{Radial stiffness}

Consider a thick disk under internal and external pressure. The radial displacement $u_{r}$ is well known:

$$
u_{r}=\frac{1-\nu}{E} \frac{P_{i} R_{i}^{2}-P_{e} R_{e}^{2}}{R_{e}^{2}-R_{i}^{2}} r+\frac{1+\nu}{E} \frac{R_{i}^{2} R_{e}^{2}}{r} \frac{P_{i}-P_{e}}{R_{e}^{2}-R_{i}^{2}}
$$

with $P_{i}, P_{e}$ respectively the internal and external pressure and $R_{i}, R_{e}$ the corresponding radii. Calling $s$ and $q$ the internal and external pressure and $c$ the external radius (the internal being obviously $b),(3)$ becomes

$$
u_{r}=\frac{1-\nu}{E_{1}} \frac{s b^{2}-q c^{2}}{c^{2}-b^{2}} r+\frac{1+\nu}{E_{1}} \frac{b^{2} c^{2}}{r} \frac{s-q}{c^{2}-b^{2}}
$$


Assuming that the external ring is radially constrained $q$ can be estimated by imposing $\left.u_{r}\right|_{r=c}=0$ :

$$
q=\frac{2 b^{2} s}{(1-\nu) c^{2}+(1+\nu) b^{2}}
$$

Thus, substituting (5) in (4) and setting $r=b$, we obtain

$$
\left.u_{r}\right|_{r=b}=\frac{b\left(c^{2}-b^{2}\right) s\left(1-\nu^{2}\right)}{\left[c^{2}(1-\nu)+b^{2}(1+\nu)\right] E_{1}}
$$

The radial displacement of an infinite plane with a hole of radius $b$ can easily be computed by taking the limit of (3) when $R_{e}$ goes to infinity and $P_{e}=0$ :

$$
\lim _{R_{e} \rightarrow \infty} u_{r}=\frac{b^{2} s(1+\nu)}{r E}
$$

Thus, by evaluating (7) when $r=b$ and posing the result equal to (6), we get the first constraint to be solved:

$$
\frac{b(1+\nu)}{E}=\frac{b\left(c^{2}-b^{2}\right)\left(1-\nu^{2}\right)}{\left[c^{2}(1-\nu)+b^{2}(1+\nu)\right] E_{1}}
$$

Note that (8) depends on two variables: Young's modulus of ring material $E_{1}$ and the outer radius of the ring $c$.

\subsection{Torsional stiffness}

The stress distribution around a hole subject to a concentrated couple is given by Muskhelishvili [7. Starting from this solution, the displacement field around

\footnotetext{
${ }^{5}$ In principle, the ring could be radially free, because load is self-balanced, but this assumption leads to physically inconsistent results (either a negative Young's modulus or $c<b$ is obtained)
} 
a hole with radius $b$ in an infinite plane is

$$
u_{\theta}=\frac{M}{2 \pi r G}
$$

where $M$ is the concentrated couple for unit thickness and $G$ the shear modulus.

By the superposition principle it is possible to compute the displacement field in the equivalent ring: assuming that it is constrained tangentially at $r=c$, the angular displacement becomes $u_{\theta}=M(c-r) /\left[2 \pi c r G_{1}\right]$, thus, the displacement of the ring when $r=b$ is

$$
\left.u_{\theta}\right|_{r=b}=\frac{(c-b) M}{2 \pi b c G_{1}}
$$

We require that the equivalent ring behaves at the internal interface as an infinite plane, thus equating (10) with 9 estimated at $b$, we get

$$
\frac{c G_{1}-(c-b) G}{b c G G_{1}}=0
$$

which constitutes the second constraint to be satisfied.

\subsection{Ring Geometry}

Solving the system of equations (8) and (11) for $E_{1}$ and $c$ gives the width and Young's Modulus of the ring:

$$
E_{1}=E \frac{2 \nu}{1+\nu} \quad c=b \frac{1+\nu}{1-\nu}
$$

The new formulation does not require calibration of the width of ring and is able to mimic both the radial and torsional behavior of an infinite plate. The counterpart is the dimension of the ring (assuming $\nu=0.3, c \simeq 1.86 b$ ) which may affect computational time. Note that the material of the external ring is actually softer than the original one because $\nu \leq 1 / 2$ for isotropic materials. 
Figure 3 compares the theoretical displacement field related to the shear loading case (both the ring formulations give exact results for the hydrostatic case) with FEM simulations performed using either infinite elements or the original and the new ring formulation. Both rings outperform the infinite element formulation, with the new one giving slightly better results. Note that they depart from the theoretical result for the $u_{\theta}$ degree of freedom far from the hole. This is not a significant problem because we are mainly interested in behavior in the near field.

\section{An Alternative Solution: Precomputed Bound- ary Conditions}

The calibrated-stiffness ring approach grants exact computation of radial displacements for the hydrostatic case and very small deviations of the same quantity in the shear-loading case. This ensures accurate calibration of the holedrilling method when using strain gauges. However, this is not enough when optical methods are used: depending on sensitivity direction, the angular and out-of-plane displacement components could be involved in computation. The accuracy of the former, although improved, is still somewhat unsatisfactory, thus a better solution is required.

Such a solution can be obtained by reconsidering why the fit-ring has been introduced: it must ensure that stiffness at $b$ matches that of an infinite plane, i.e. it must ensure that the displacement at $b$ will be the same of the theoretical plane for a given external load. By reversing the reasoning and considering the linear stress-strain relation, if a displacement corresponding to the theoretical one is imposed on boundaries, a stress field corresponding to the theoretical solution will result inside the plate. 
In the general case (e.g. blind-hole) the exact displacements are unknown, but if we are far enough from the hole we can assume that the displacements will be given by (1a) $-1 \mathrm{c}$ ) by the Saint Venant principle, providing that we scale the stress level by the ratio of the loaded depth to the thickness of the plate. Thus, recovering the theoretical solution simply requires prescribing the $\left.u_{r}\right|_{r=b}$, $\left.u_{\theta}\right|_{r=b},\left.u_{z}\right|_{r=b}$ resulting from (1) at the external boundary and performing the simulation 6 .

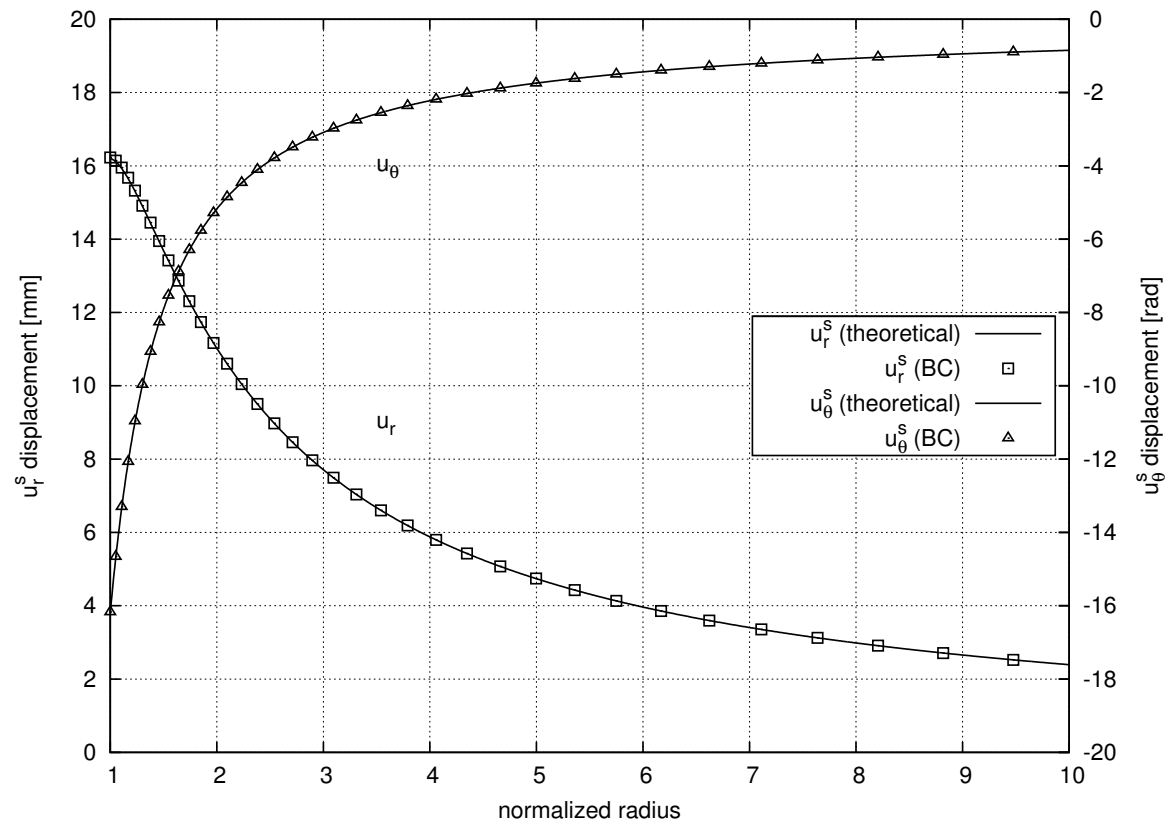

Figure 4: Comparing the theoretical result (hole-through case) and FEM solution with assigned external displacements (shear-loading case). A similar performance was obtained in the hydrostatic case.

Figure 4 compares the theoretical displacements related to the shear case (continuous line) with FEM results obtained using the proposed approach. The agreement is excellent. The same can be stated for the hydrostatic case (not

\footnotetext{
${ }^{6}$ Note that if a semi-analytical solution is used (i.e. an axial-symmetric computation expanded in Fourier series), the $\cos (2 \theta)$ and $\sin (2 \theta)$ terms are implicit in the expansion, thus $u_{r}$ has to be evaluated at $\theta=0$ whereas $u_{\theta}$ has to be computed assuming $\theta=\pi / 4$.
} 
shown).

\section{Conclusions}

In this work, implementation of the external boundary conditions for holedrilling calibration is discussed and shows that the standard solution (fully constrained or free external boundary) may lead to some inaccuracies, in particular when optical methods are taken into account.

To solve this problem, two solutions are proposed: either by adding an external ring, whose stiffness matches the radial and torsional behavior of an infinite plane, or imposing precomputed displacements at boundary nodes. The former grants that the system will always behave correctly, but requires a large ring (thus affecting computational time significantly); the latter ensures exact results over the full field, providing the Saint Venant principle is valid and the stiffness of the core does not significantly affect the behavior of the system. Because this requirement can easily be satisfied, the latter solution should always be preferred, in particular when optical methods and reverse calibration procedures are employed to estimate residual stress.

\section{Acknowledgements}

I am grateful to Prof. G. S. Schajer with whom I discussed some of the topics investigated in this paper. He provided me insightful comments and constructive criticisms at different stages of my work. Any error contained herein, naturally, remain my responsibility. 


\section{References}

[1] ASTM E837-08e1. Standard test method for determining residual stresses by the hole-drilling strain-gage method. , American Society for Testing and Materials, West Conshohocken, PA, 2008. doi:10.1520/E0837-08E01.

[2] Antonio Baldi. A new analytical approach for hole drilling residual stress analysis by full field method. Journal of Engineering Materials and Technology, 127(2):165-169, 2005.

[3] G. S. Schajer and M. Steinzig. Full-field calculation of hole drilling residual stresses from electronic speckle pattern interferometry data. Experimental Mechanics, 45(6):526-532, 2005.

[4] G. S. Schajer. Advances in hole-drilling residual stress measurements. Experimental Mechanics, 50(2):159-168, 2010.

[5] D. V. Nelson, A. Makino, and E. A. Fuchs. The holographic-hole drilling method for residual stress determination. Optics and Lasers in Engineering, 27:3-23, 1997.

[6] Olgierd Cecil Zienkiewicz and Robert Leroy Taylor. The Finite Element Method: the Basis, volume 1, chapter 9, pages 229-234. ButterworthHeinemann, $5^{\text {th }}$ edition, 2000. ISBN 0-7506-5049-4.

[7] N. I. Muskhelishvili. Some Basic Problems of the Mathematical Theory of Elasticity, chapter 9, pages 210-211. P. Noordhoff Ltd, Groningen, The Netherlands, translated from fourth, corrected and augmented russian edition, 1963. Translated from the Russian by J.R.M. Radok. 